\title{
Probing gaugino coannihilation with displaced vertex searches
}

\author{
Natsumi Nagata*† \\ William I. Fine Theoretical Physics Institute, School of Physics and Astronomy, \\ University of Minnesota, Minneapolis, Minnesota 55455, USA \\ E-mail: nagat006@umn. edu
}

Bino-like dark matter in supersymmetric theories tends to suffer from over-production. This problem can be evaded if there is another gaugino which has a mass close to the bino mass so that it enhances the annihilation rate of bino dark matter through coannihilation. We study such possibilities in high-scale supersymmetric models, and show that searches for long-lived particles with displaced vertices can test the bino-gaugino coannihilation scenario in the forthcoming LHC experiments.

The 11th International Workshop Dark Side of the Universe 2015

14-18 December 2015

Kyoto, Japan

\footnotetext{
* Speaker.

${ }^{\dagger}$ Based on work in collaboration with Hidetoshi Otono and Satoshi Shirai $[1,2]$.
} 


\section{Introduction}

Despite plenty of evidence for the presence of dark matter (DM), we have almost no idea what it is. Weakly-interacting massive particles (WIMPs) are the most promising class of candidates for DM, since the observed DM density can naturally be explained by their thermal relic abundance. Many beyond-the-Standard-Model theories predict WIMPs as their inevitable ingredients. Among them, the minimal supersymmetric Standard Model (MSSM) offers a very attractive candidate for WIMP DM: the lightest neutralino, which is an electrically neutral and colorless fermion composed of a linear combination of bino $\widetilde{B}$ (the superpartner of the $\mathrm{U}(1)_{Y}$ gauge field), the neutral wino $\widetilde{W}_{3}$ (winos $\widetilde{W}_{a}, a=1,2,3$, are the superpartners of the $\mathrm{SU}(2)_{L}$ gauge bosons), and higgsinos $H_{u}$ and $H_{d}$ (the superpartners of the Higgs boson). The stability of the neutralino DM is assured by the $R$-parity if it is the lightest supersymmetric (SUSY) particle (LSP) [3].

On the other hand, the recent LHC results have imposed stringent constraints on TeV-scale physics. Regarding SUSY models, multi-jets plus missing energy searches restrict masses of SUSY particles, especially those of squarks and gluino [4]. In addition, the mass of the Standard Model (SM) Higgs boson, $m_{h} \simeq 125 \mathrm{GeV}$ [5], implies that SUSY partners are heavy, since large quantum corrections to the Higgs mass by heavy stops are required in order to achieve the observed Higgs mass in the MSSM. Consequently, the early LHC results might indicate that the SUSY scale is much higher than the electroweak scale, contrary to the expectation before the LHC run.

Considering this situation, the questions we would like to address here are the following. Is the neutralino DM still promising? Can we probe the neutralino DM in future experiments? To answer these questions, it is important to list up the present allowed parameter regions for the neutralino DM, and study their phenomenological consequences.

The constrained MSSM (CMSSM) is a widely studied benchmark scenario for SUSY models. Recently, a parameter scan in the CMSSM was performed in Ref. [6]. This result shows that the correct DM relic abundance can be obtained only in specific parameter regions (see Ref. [7] for more detailed discussions). Most of the parameter points will be probed in future LHC experiments, DM searches, or proton decay experiments $[6,7,8]$.

On the other hand, SUSY models with a high SUSY-breaking scale, as high as 100-1000 TeV [9], have attracted wide attention recently, especially after the Higgs discovery [10]. These models are based on the assumption that there is no singlet SUSY-breaking field in the SUSY breaking sector, which couples to the MSSM sector via a generic form of the Kähler potential. In this case, all of the scalar particles except the SM Higgs boson have masses similar to the gravitino mass, $m_{3 / 2}$. The supersymmetric higgsino mass $\mu$ is also expected to be around $m_{3 / 2}$, though it depends on models. In what follows, we assume that a typical scale of soft masses, $\widetilde{m}$ and the higgsino mass $\mu$ is $\mathscr{O}\left(m_{3 / 2}\right){ }^{1}$ The gaugino masses are, on the other hand, induced only by quantum corrections, and thus suppressed by a loop factor compared with $m_{3 / 2}$. Such quantum effects include the anomaly mediation $[15,16]$ and threshold corrections by heavy Higgs bosons $[16,17]$, extra vector-like multiplets whose masses are $\mathscr{O}\left(m_{3 / 2}\right)[18]$, or particles appearing around

\footnotetext{
${ }^{1}$ In models where $\mu \ll m_{3 / 2}$, which can be achieved by means of a certain symmetry such as the Peccei-Quinn symmetry [11], the neutral higgsino can be a viable dark matter candidate. See Refs. [12, 13] for concrete examples for the realization of the higgsino LSP. Regarding the phenomenology of the higgsino DM scenario, see Ref. [14] and references therein.
} 


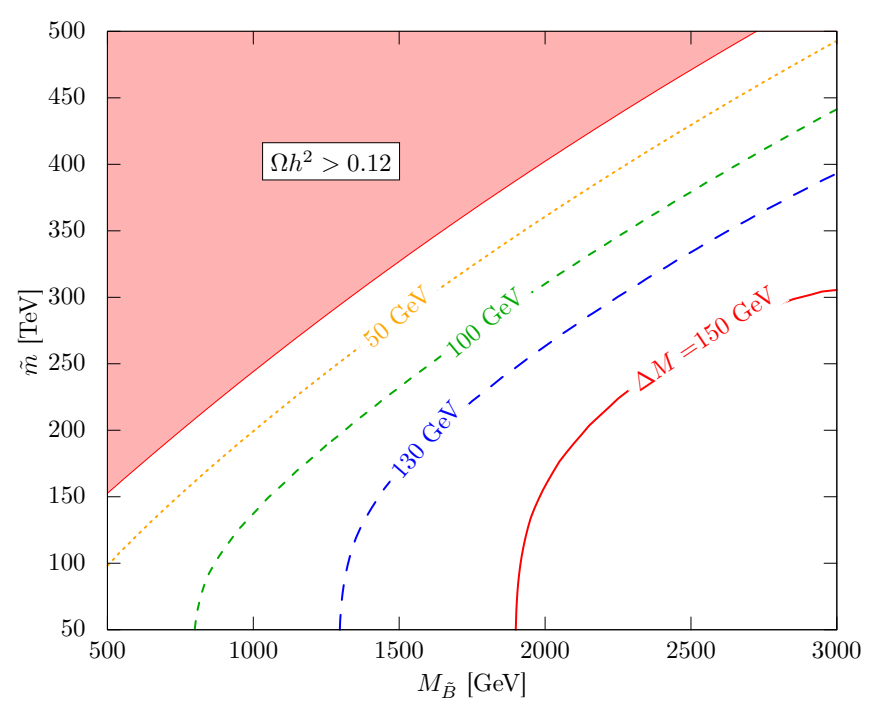

Figure 1: Contours for the mass difference $\Delta M$ which achieves $\Omega_{\mathrm{DM}} h^{2}=0.12$.

the grand unification scale [19]. We therefore expect that the LSP is one of the gauginos, though their mass spectrum depends on sizes of these quantum corrections.

In this case, there are two DM candidates; one is the neutral wino and the other is bino. The wino DM is known to be a good DM candidate. Its thermal relic abundance agrees to the observed DM density if the wino mass is $\simeq 3 \mathrm{TeV}$ [20]. This DM can be probed in both direct [21] and indirect [22] DM searches. On the other hand, the bino DM often suffers from over production due to its small annihilation cross section. Thus, some additional mechanisms are required to reduce its thermal relic. In the present scenario, we can use coannihilation [23] with either gluinos $[24,25,26,1]$ or winos $[27,25,2]$. We discuss these two scenarios in this article.

Both of these scenarios require the lightest and the next-to-lightest particles to be highly degenerate in mass, which makes it difficult to probe these scenarios with traditional LHC search methods, since decay products of the next-to-lightest SUSY particle (NLSP) tend to be too soft. Furthermore, it is also hard to detect the bino DM in DM searches since its interactions are extremely weak. It turns out, however, that in both of these scenarios the NLSP becomes long-lived, which results in specific signatures at collider experiments. Thanks to this feature, we can probe these coannihilation scenarios using displaced vertex (DV) searches [1, 2, 28], as we will see below.

\section{Bino-gluino coannihilation}

First we consider the case in which gluino is degenerate with bino in mass so that the relic abundance of the bino DM can be sufficiently reduced because of the coannihilation mechanism. In order for the coannihilation to work effectively, however, chemical equilibrium between bino and gluino should be maintained until the time when the bino DM is decoupled from thermal bath $[26,1]$. This requires the transition rate of bino into gluino, $\Gamma(\widetilde{B} q \rightarrow \widetilde{g} q)$, be much larger than the Hubble expansion rate, $H$. This transition is induced by the squark exchange diagrams and its amplitude is suppressed by $\widetilde{m}^{-2}$. Therefore, this requirement gives an upper bound on $\widetilde{m}$. In Fig. 1, 


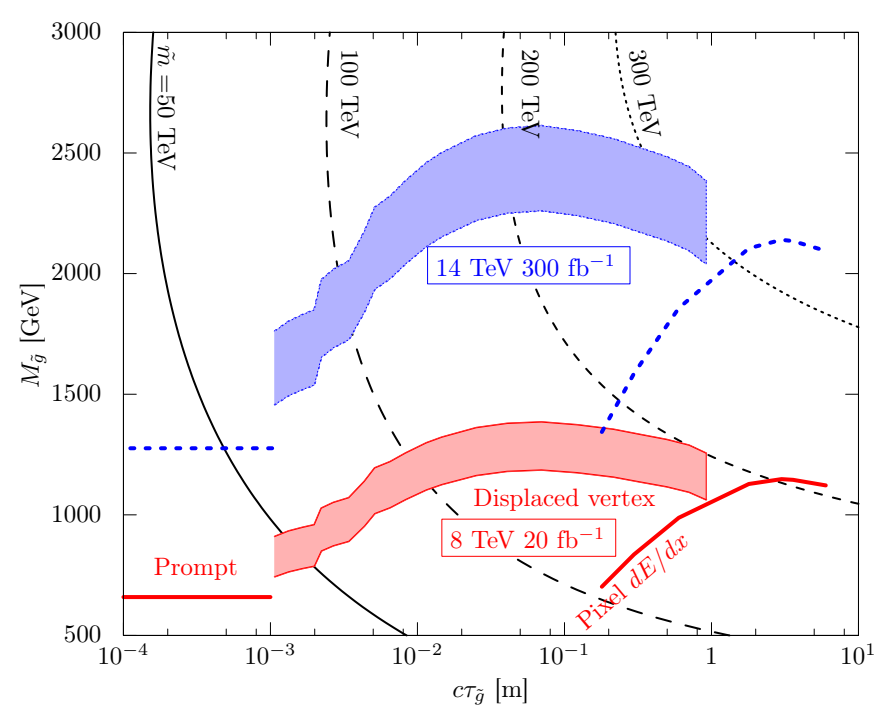

Figure 2: Current limits (red solid) and future prospects (blue dashed) of long-lived gluino searches [1].

we show contour lines for the required mass difference between bino and gluino, $\Delta M \equiv M_{\widetilde{g}}-M_{\widetilde{B}}$ ( $M_{\widetilde{g}}$ and $M_{\widetilde{B}}$ are the masses of gluino and bino, respectively), to explain the observed DM density $\Omega_{\mathrm{DM}} h^{2}=0.12$ with the thermal relic of the bino LSP. In the pink shaded area, bino is over produced because the transition rate $\Gamma(\widetilde{B} q \rightarrow \widetilde{g} q)$ is too small. From this figure we find that the bino-gluino coannihilation scenario requires $\Delta M \simeq 100 \mathrm{GeV}$.

With such a small $\Delta M$, gluinos tend to be long-lived. Gluinos decay into SM quarks and a bino again via the squark exchange. The gluino decay length is roughly given by

$$
c \tau_{\widetilde{g}} \simeq\left(\frac{\Delta M}{100 \mathrm{GeV}}\right)^{-5}\left(\frac{\widetilde{m}}{100 \mathrm{TeV}}\right)^{4} \mathrm{~cm},
$$

and thus in the bino-gluino coannihilation scenario we expect $\mathscr{O}(1) \mathrm{cm}$ decay length, which can be a good target of the DV searches as we will discuss below.

The ATLAS collaboration has searched for DVs in the inner detector [29], and thus this search is sensitive to decay lengths of $1 \mathrm{~mm} \lesssim c \tau \lesssim 1 \mathrm{~m}$. Among the several search categories in Ref. [29], the DV plus missing transverse energy $\left(E_{\mathrm{T}}^{\text {miss }}\right)$ search is most sensitive to the present setup. Using the data collected at the LHC $8-\mathrm{TeV}$ run with an integrated luminosity of $20.3 \mathrm{fb}^{-1}$, the ATLAS collaboration has searched for such events, and has observed no signal. This result then gives a lower limit on $M_{\widetilde{g}}$ [29]; for instance, if the neutralino LSP mass is $100 \mathrm{GeV}$, a lower limit on $M_{\widetilde{g}}$ is given as $M_{\tilde{g}} \gtrsim 1.5 \mathrm{TeV}$ for $c \tau_{\widetilde{g}} \simeq 10 \mathrm{~cm}$. We however note that we cannot directly apply this result to the bino-gluino coannihilation case, since in this case we need to take into account the fact that $\Delta M$ is as small as $100 \mathrm{GeV}$. To that end, we simulate the reduction of trigger efficiency due to the small $\Delta M$ by using HERWIG6 [30] and AcerDET [31], and reinterpret the ATLAS result. Here, we require $E_{\mathrm{T}}^{\text {miss }}>100 \mathrm{GeV}$ for the $8-\mathrm{TeV}$ run. For the estimation of the future prospects, we assume the $14 \mathrm{TeV}$ run with an integrated luminosity of $300 \mathrm{fb}^{-1}$, and require $E_{\mathrm{T}}^{\text {miss }}>200 \mathrm{GeV}$. In Fig. 2 we show the current constraints and future prospects for the long-lived gluino searches in red solid and blue dashed lines, respectively. For $c \tau_{\widetilde{g}}<1 \mathrm{~mm}$, we use the ordinary limit from the gluino 


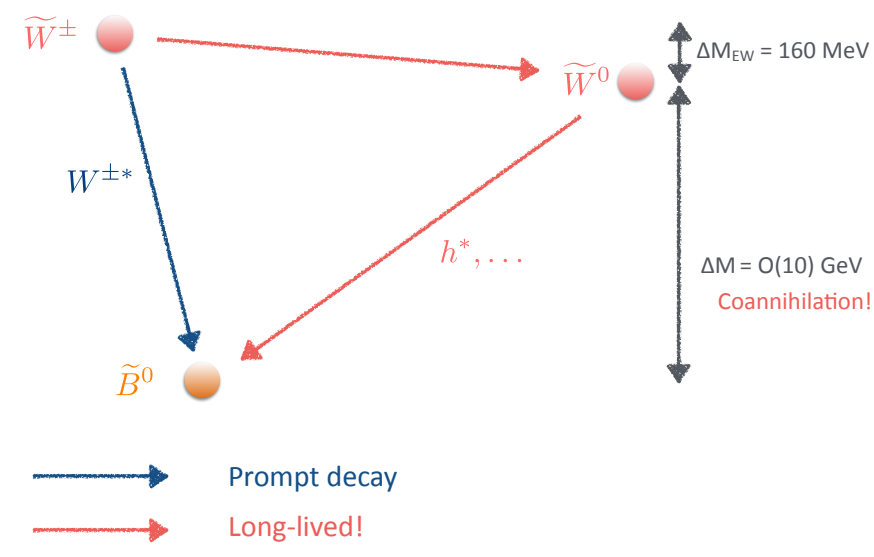

Figure 3: Mass spectrum and decay chains of the bino-wino system.

search based on jets plus $E_{\mathrm{T}}^{\text {miss }}$ only [32]. For $10^{-3} \mathrm{~m}<c \tau_{\widetilde{g}}<1 \mathrm{~m}$, we show our estimate based on the ATLAS DV search [29], while for $c \tau_{\widetilde{g}} \gtrsim 10^{-1} \mathrm{~m}$, we plot the limit from the $R$-hadron search [33]. To estimate the uncertainty of our evaluation, we vary reconstruction efficiency for DVs between $20-100 \%$ of that in Ref. [29], which is shown in the bands in the figure. We also show the black contour lines along which the observed DM density is obtained with several values of $\widetilde{m}$. This figure shows that a wide region of parameter space favored by the bino-gluino coannihilation scenario can be probed with the DV search. In particular, its sensitivity is found to be better than those of the ordinary gluino searches based on multi-jets plus $E_{\mathrm{T}}^{\mathrm{miss}}$.

\section{Bino-wino coannihilation}

Next, we discuss the case of the bino-wino coannihilation scenario [2]. It turns out that the NLSP again tends to be long-lived over the parameter region favored by the bino-wino coannihilation scenario. The relevant mass spectrum is shown in Fig. 3. As we will see below, the bino-wino coannihilation scenario requires the bino-wino mass difference, $\Delta M$, to be $\mathscr{O}(10) \mathrm{GeV}$. A neutral wino can decay into the bino LSP via mixing with higgsinos. Therefore, the decay rate is suppressed if $\mu$ is very large. It is found that in this case its dominant decay mode is induced by an off-shell Higgs boson emission. Thus, the amplitude is also suppressed by small Yukawa couplings. Notice that the $Z$ boson exchange process is sub-dominant in this setup. The decoupling features of these decay amplitudes with respect to large $\mu$ is studied in Ref. [2] using the effective field theoretical approach. Moreover, there is an additional suppression factor due to the small mass difference $\Delta M$. For these reasons, the decay length of the neutral wino NLSP becomes about $1 \mathrm{~cm}-1 \mathrm{~m}$, which can again be probed with DV searches [2, 34]. In Fig. 4(a), we show the decay length of the neutral wino in the black solid (red dashed) contour lines in the $M_{\widetilde{B}}-\Delta M$ plane, where we set $\mu=100 \mathrm{TeV}$ and $\tan \beta=1(\mu=25 \mathrm{TeV}$ and $\tan \beta=30)$. The blue dashed line corresponds to the parameter points where the observed DM density is explained by the thermal relic of the bino LSP. As can be seen, the neutral wino has a decay length of $\gtrsim 1 \mathrm{~cm}$ over the parameter region 


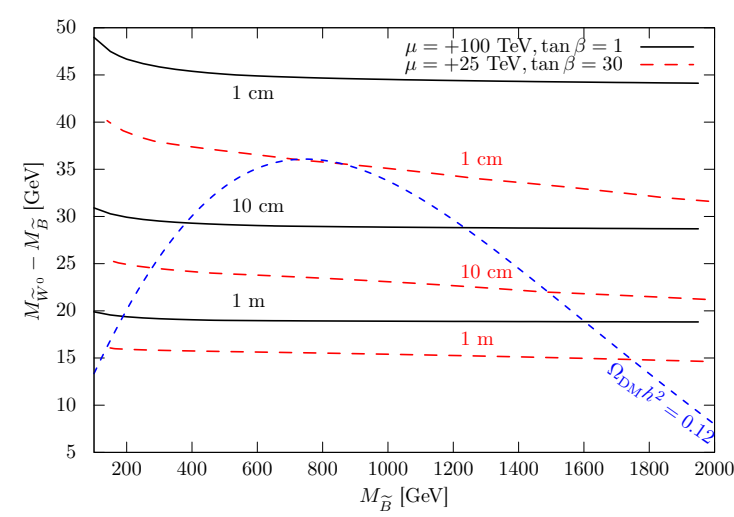

(a) Decay length

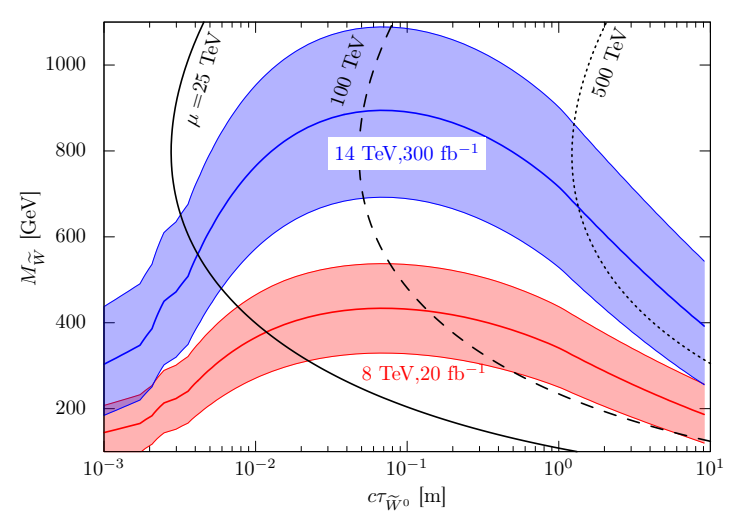

(b) Constraints and prospects

Figure 4: (a) The decay length of the neutral wino. (b) The present limit (red) and future prospects (blue) of the long-lived neutral wino search [2].

motivated by the bino-wino coannihilation scenario. On the other hand, a charged wino, which has a slightly larger mass than a neutral wino because of the radiative corrections, decays into bino promptly through an off-shell emission of the $W$ boson as long as $|\mu|<\mathscr{O}\left(10^{4}\right) \mathrm{TeV}$.

Similarly to the previous case, we reinterpret the ATLAS DV search result [29] to study the current constraints and future prospects of the bino-wino coannihilation scenario. For this purpose, we use the program packages Madgraph 5 [35], Pythia6 [36], and Delphes 3 [37] for the estimation of the acceptance rate, while we use Prospino2 [38] for the computation of the cross sections. In this analysis, we have dropped the $P_{\mathrm{T}}$ condition for DVs adopted in Ref. [29], to optimize this search for the bino-wino coannihilation scenario. In this case, a large $E_{\mathrm{T}}^{\text {miss }}$ results from the back reaction of initial state radiations. We then obtain the current constraints and future prospects of the long-lived neutral wino search, which is shown in Fig. 4(b) in red and blue lines, respectively. Here, we set $\Delta M=30 \mathrm{GeV}$ and $\tan \beta=2$. The bands represent the uncertainties of our estimation, where we vary the acceptance rate by a factor of three. The black contour lines correspond to the parameter points where the correct DM density is obtained for $\mu=25,100$, and $500 \mathrm{TeV}$, respectively (from left to right). This figure shows that a wino with a mass of 400 (800) $\mathrm{GeV}$ can be probed at the 8 (14) TeV LHC in the case of the bino-wino coannihilation scenario.

\section{Summary}

In this article, we discuss possibilities of bino DM in the high-scale SUSY scenario. In this case, the observed DM density can be explained if gluino or wino is degenerate with the bino LSP in mass. Because of the mass degeneracy as well as large masses of the exchanged particles in the decay processes, the NLSP tends to have long lifetime, which can be probed with DV searches. For the bino-gluino coannihilation scenario, the $14 \mathrm{TeV}$ LHC run can reach a gluino mass of $\sim$ $2 \mathrm{TeV}$, while for the bino-wino coannihilation scenario, the expected reach for the wino mass is $\sim 800 \mathrm{GeV}$. 


\section{Acknowledgments}

The author thanks H. Otono and S. Shirai for collaboration. This work was supported by the DOE grant DE-SC0011842 at the University of Minnesota.

\section{References}

[1] N. Nagata, H. Otono and S. Shirai, Phys. Lett. B 748, 24 (2015) [arXiv: 1504.00504 ].

[2] N. Nagata, H. Otono and S. Shirai, JHEP 1510, 086 (2015) [arXiv: 1506.08206 ].

[3] H. Goldberg, Phys. Rev. Lett. 50, 1419 (1983); J. R. Ellis, J. S. Hagelin, D. V. Nanopoulos, K. A. Olive and M. Srednicki, Nucl. Phys. B 238, 453 (1984).

[4] G. Aad et al. [ATLAS Collaboration], JHEP 1510, 054 (2015) [arXiv: 1507 . 05525]; V. Khachatryan et al. [CMS Collaboration], JHEP 1505, 078 (2015) [arXiv: 1502.04358 ].

[5] G. Aad et al. [ATLAS and CMS Collaborations], Phys. Rev. Lett. 114, 191803 (2015) [arXiv:1503.07589].

[6] E. A. Bagnaschi et al., Eur. Phys. J. C 75, 500 (2015) [arXiv:1508.01173].

[7] K. A. Olive, arXiv:1604.07336.

[8] J. Ellis, J. L. Evans, F. Luo, N. Nagata, K. A. Olive and P. Sandick, Eur. Phys. J. C 76, 8 (2016) [arXiv:1509.08838].

[9] J. D. Wells, hep-ph/ 0306127 ; N. Arkani-Hamed and S. Dimopoulos, JHEP 0506, 073 (2005) [hep-th/ 040515 9]; G. F. Giudice and A. Romanino, Nucl. Phys. B 699, 65 (2004) Erratum: [Nucl. Phys. B 706, 487 (2005)] [hep-ph/ 0406088 ]; N. Arkani-Hamed, S. Dimopoulos, G. F. Giudice and A. Romanino, Nucl. Phys. B 709, 3 (2005) [hep-ph/ 0409232 ]; J. D. Wells, Phys. Rev. D 71, 015013 (2005) [hep-ph/ 0411041 ].

[10] L. J. Hall and Y. Nomura, JHEP 1201, 082 (2012) [arXiv:1111.4519]; M. Ibe and T. T. Yanagida, Phys. Lett. B 709, 374 (2012) [arXiv:1112.2462]; M. Ibe, S. Matsumoto and T. T. Yanagida, Phys. Rev. D 85, 095011 (2012) [arXiv: 1202 . 2253]; A. Arvanitaki, N. Craig, S. Dimopoulos and G. Villadoro, JHEP 1302, 126 (2013) [arXiv: 1210 . 0555]; L. J. Hall, Y. Nomura and S. Shirai, JHEP 1301, 036 (2013) [arXiv: 1210.2395 ]; N. Arkani-Hamed, A. Gupta, D. E. Kaplan, N. Weiner and T. Zorawski, arXiv:1212.6971; J. L. Evans, M. Ibe, K. A. Olive and T. T. Yanagida, Eur. Phys. J. C 73, 2468 (2013) [arXiv: 1302 . 5346 ].

[11] R. D. Peccei and H. R. Quinn, Phys. Rev. Lett. 38, 1440 (1977).

[12] K. J. Bae, H. Baer and H. Serce, Phys. Rev. D 91, 015003 (2015) [arXiv: 1410 . 7500 ].

[13] J. L. Evans, M. Ibe, K. A. Olive and T. T. Yanagida, Phys. Rev. D 91, 055008 (2015) [arXiv:1412.3403].

[14] N. Nagata and S. Shirai, JHEP 1501, 029 (2015) [arXiv: 1410 . 4549].

[15] L. Randall and R. Sundrum, Nucl. Phys. B 557, 79 (1999) [hep-th/ 9810155 ].

[16] G. F. Giudice, M. A. Luty, H. Murayama and R. Rattazzi, JHEP 9812, 027 (1998) [hep-ph/9810442].

[17] D. M. Pierce, J. A. Bagger, K. T. Matchev and R. j. Zhang, Nucl. Phys. B 491, 3 (1997) [hep-ph/9606211]. 
[18] A. Pomarol and R. Rattazzi, JHEP 9905, 013 (1999) [hep-ph/99034 48]; A. E. Nelson and N. J. Weiner, hep-ph/ 0210288 ; K. Hsieh and M. A. Luty, JHEP 0706, 062 (2007) [hep-ph/0604256]; A. Gupta, D. E. Kaplan and T. Zorawski, JHEP 1311, 149 (2013) [arXiv:1212.6969]; K. Nakayama and T. T. Yanagida, Phys. Lett. B 722, 107 (2013) [arXiv:1302.3332]; K. Harigaya, M. Ibe and T. T. Yanagida, JHEP 1312, 016 (2013) [arXiv:1310.0643]; J. L. Evans and K. A. Olive, Phys. Rev. D 90, 115020 (2014) [arXiv:1408.5102].

[19] J. Hisano, H. Murayama and T. Goto, Phys. Rev. D 49, 1446 (1994).

[20] J. Hisano, S. Matsumoto, M. Nagai, O. Saito and M. Senami, Phys. Lett. B 646, 34 (2007) [hep-ph/0610249].

[21] J. Hisano, K. Ishiwata and N. Nagata, JHEP 1506, 097 (2015) [arXiv: 1504.00915 ].

[22] B. Bhattacherjee, M. Ibe, K. Ichikawa, S. Matsumoto and K. Nishiyama, JHEP 1407, 080 (2014) [arXiv:1405.4914].

[23] K. Griest and D. Seckel, Phys. Rev. D 43, 3191 (1991).

[24] S. Profumo and C. E. Yaguna, Phys. Rev. D 69, 115009 (2004) [hep-ph/ 0402208 ]; D. Feldman, Z. Liu and P. Nath, Phys. Rev. D 80, 015007 (2009) [arXiv:0905.1148]; A. De Simone, G. F. Giudice and A. Strumia, JHEP 1406, 081 (2014) [arXiv: 1402 . 6287].

[25] K. Harigaya, K. Kaneta and S. Matsumoto, Phys. Rev. D 89, 115021 (2014) [arXiv: 1403.0715$].$

[26] J. Ellis, F. Luo and K. A. Olive, JHEP 1509, 127 (2015) [arXiv: 1503.07142 ]; J. Ellis, J. L. Evans, F. Luo and K. A. Olive, JHEP 1602, 071 (2016) [arXiv: 1510 . 03498].

[27] H. Baer, T. Krupovnickas, A. Mustafayev, E. K. Park, S. Profumo and X. Tata, JHEP 0512, 011 (2005) [hep-ph/ 0511034 ]; N. Arkani-Hamed, A. Delgado and G. F. Giudice, Nucl. Phys. B 741, 108 (2006) [hep-ph/ 0601041 ]; M. Ibe, A. Kamada and S. Matsumoto, Phys. Rev. D 89, 123506 (2014) [arXiv:1311.2162].

[28] H. Otono, PoS EPS -HEP2015, 182 (2015) [arXiv: 1509.03613 ].

[29] G. Aad et al. [ATLAS Collaboration], Phys. Rev. D 92, 072004 (2015) [arXiv: 1504.05162 ].

[30] G. Corcella, I. G. Knowles, G. Marchesini, S. Moretti, K. Odagiri, P. Richardson, M. H. Seymour and B. R. Webber, JHEP 0101, 010 (2001) [hep-ph/ 0011363 ]; G. Corcella, I. G. Knowles, G. Marchesini, S. Moretti, K. Odagiri, P. Richardson, M. H. Seymour and B. R. Webber, hep-ph/0210213.

[31] E. Richter-Was, hep-ph/0207355.

[32] S. Chatrchyan et al. [CMS Collaboration], JHEP 1406, 055 (2014) [arXiv: 1402 . 4770]; G. Aad et al. [ATLAS Collaboration], JHEP 1409, 176 (2014) [arXiv: 1405 . 7875].

[33] G. Aad et al. [ATLAS Collaboration], Eur. Phys. J. C 75, 407 (2015) [arXiv: 1506.05332 ].

[34] K. Rolbiecki and K. Sakurai, JHEP 1511, 091 (2015) [arXiv: 1506.08799 ].

[35] J. Alwall, M. Herquet, F. Maltoni, O. Mattelaer and T. Stelzer, JHEP 1106, 128 (2011) [arXiv:1106.0522].

[36] T. Sjostrand, S. Mrenna and P. Z. Skands, JHEP 0605, 026 (2006) [hep-ph/ 0603175 ].

[37] J. de Favereau et al. [DELPHES 3 Collaboration], JHEP 1402, 057 (2014) [arXiv: 1307.6346 ].

[38] W. Beenakker, R. Hopker and M. Spira, hep-ph/9611232. 